\title{
Screening family planning needs: an operations research project in Guatemala
}

\author{
Francisco Mendez ${ }^{1}$, Felipe Lopez ${ }^{2}$, Carlos Brambila*3 and \\ Marianne Burkhart ${ }^{4}$
}

\begin{abstract}
Address: ${ }^{1}$ At the time of the study: Guatemala office, Population Council, Guatemala City, Guatemala. Presently: Regional management, CARE, Guatemala City, Guatemala, ${ }^{2}$ At the time of the study, Guatemala office, Population Council, Guatemala City, Guatemala. Presently: Training department, PROREDES Project, Guatemala City, Guatemala, ${ }^{3}$ At the time of the study, Guatemala office, Population Council. Guatemala City, Guatemala. Presently: School of Public Administration and Public Policies, Instituto Tecnológico de Monterrey, Mexico City, Mexico and ${ }^{4}$ At the time of the study, Guatemala office, Population Council, Guatemala City, Guatemala. Presently: Independent consultant, Seattle, Washington, U.S.A
\end{abstract}

Email: Francisco Mendez - fmendez@care.org.gt; Felipe Lopez - flopez@proredes.org.gt; Carlos Brambila* - carlos.brambila@itesm.mx; Marianne Burkhart - mcburkhart3@juno.com

* Corresponding author

Published: 06 May 2004

BMC International Health and Human Rights 2004, 4:2

This article is available from: http://www.biomedcentral.com/I472-698X/4/2

(C) 2004 Mendez et al; licensee BioMed Central Ltd. This is an Open Access article: verbatim copying and redistribution of this article are permitted in al media for any purpose, provided this notice is preserved along with the article's original URL.
Received: 27 October 2003

Accepted: 06 May 2004

\begin{abstract}
Background: Public sector health care providers in rural Guatemala have infrequently offered family planning information and services in routine visits. This operations research project tested a strategy to modify certain practices that prevent health workers from proactively screening clients' needs and meeting them.

Methods: The research design was quasi-experimental with a pretest-posttest-follow-up comparison group design. Health districts, which comprise health centers and posts, were purposively assigned to intervention or comparison groups to assure comparability of the two groups. The strategy was based on a job-aid designed to guide health workers in screening clients' reproductive intentions and family planning needs, help them to offer contraceptive methods if the woman expressed interest, and facilitate the provision of the method chosen at the time of the visit. The strategy was implemented at intervention sites during a period of six months. Upon completion of post-intervention measurements, the strategy was scaled up to the comparison sites, and a follow-up assessment was conducted nine months later. Results were evaluated by conducting three rounds of exit interviews with women exposed to the risk of unwanted pregnancy.

Results: Study results showed a two to five-fold increase in providers' screening of clients' reproductive intentions. The proportion of clients who received information about contraceptives increased from $8 \%$ at the baseline to $42 \%$ immediately post-intervention, and $36 \%$ at the follow-up survey. The intervention also proved successful in improving the role service providers play in offering women a chance to ask questions and assisting women in making a selection. The proportion of women who received a method, referral or appointment increased and remained high in the intervention group, although no change was seen in the comparison group after their participation in the strategy.

Conclusion: The easy-to-use job aid developed for this project proved useful for screening clients' needs and reducing providers' reluctance to discuss family planning with clients and offer contraceptive services. Such family planning screening devices can be useful in traditional settings where both providers and clients shy away from discussing family planning issues.
\end{abstract}




\section{Background}

Although substantial research has been undertaken to understand medical barriers to family planning service provision, [1-3] few operational interventions have demonstrated strategies that successfully overcome constraints imposed by providers. In traditional settings, the most important medical barrier is often the providers' reluctance to ask women about their reproductive intentions and discuss contraceptive methods openly. Because of cultural, personal or institutional constraints, many service providers deliberately limit information and services.

Based on previous diagnostic studies about medical barriers $[4,5]$ researchers and policy makers in Guatemala concluded that providers routinely constrained information about family planning and lacked skills to select and organize the questions they asked and information they provided. If a woman did not state that her visit was for family planning, the provider did not proactively explore her reproductive intentions. When a woman did express interest, health care workers often provided information that had no relation to the needs and interests of the client. Inability to ask the right questions and respond with appropriate information and services led providers to the conclusion that providing family planning required too much time. Through discussion of such results, policy makers realized that service providers constrained the extent to which they offered services.

Nonexistent and inefficient screening resulted in creating a barrier for many rural women who wanted to avoid a birth in the near future. Most people in Guatemala visit health posts and centers in the morning, arriving on a very early bus from the countryside so they have time to do their marketing as well as seek health care. Commonly the last return bus leaves early in the afternoon. Health care workers routinely asked women who requested family planning to return in the afternoon when there were fewer patients to be seen. Although this was a seemingly simple request, many women found it difficult or impossible to return. Rural women are often very shy about bringing up an interest in family planning and may not have sufficient courage to do so twice in one day, while others must catch the last bus home, which in many cases means leaving town in the early afternoon. Women need to be screened and receive information and a method of their choice in the morning visit, and this requires that health care workers be able to conduct a screening efficiently and in a manner oriented to the woman's individual needs.

Researchers informed policy makers about job-aids that have proven useful in other settings in enabling service providers to screen for a woman's reproductive intentions and her contraceptive needs [6,7]. Such background research prompted decision makers to request a job aid that would help service providers to assure that clients have a window of opportunity to ask questions about contraceptive methods and discuss family planning issues with qualified personnel. This is particularly important for indigenous women who shy away from requesting these services and discussing family planning issues with Ministry of Health personnel, who are generally not indigenous.

\section{Methods \\ Program setting}

The Guatemalan population can be divided into two groups of approximately equal size: the western-oriented, primarily urban, Spanish-speaking Ladinos and the indigenous Mayan inhabitants, who comprise 23 officially recognized, distinct cultural and linguistic groups. Historically, political and economic power has been held by the upper classes of the Ladino population, and the Mayans have been marginalized from virtually all aspects of national life.

The inequality between Ladinos and Mayans is clearly marked when national health statistics are broken down by ethnicity. The national infant mortality rate for the entire population has been estimated to be 51 per thousand live births, but in many Mayan communities the rate may be twice the national average. According to demographic and health surveys, the Contraceptive Prevalence Rate increased from 32\% in 1995 [8] to $38 \%$ in 1998/99 $[9,10]$ and $43 \%$ in 2002 [11]. However, according to results of the 2002 National Maternal and Child Health Survey, the Contraceptive Prevalence Rate is $35 \%$ in rural areas and as low as $16 \%$ among indigenous populations.

Education and literacy rates are similarly disadvantageous for the Mayan population, particularly among women, which makes the expansion of information about health care difficult. Fully $31 \%$ of the Mayan population is nonliterate, one of the highest rates in Latin America [10]. Non-literacy is an obstacle to individual and family development, with repercussions on health indicators.

In 1998 the Policy Project conducted a diagnostic study of the quality of services provided at health centers and posts in Guatemala [4]. The study showed poor provider-client interpersonal relations, poor technical competence of providers, widespread misconceptions among both providers and community members about the safety and risks of contraceptives, inability of many providers to speak the Mayan language of their clients, and other structural problems. Considering that other programs were addressing some of these barriers, Ministry of Health decision makers and researchers concluded that one of the most important barriers amenable to improvement was the inability of providers to structure a screening and provide services in 
a way that is both efficient and oriented to the needs of the individual client.

\section{Intervention}

The purpose of the intervention was to modify specific practices that prevent health workers from screening clients' needs, providing information, and delivering family planning services at the time of the visit. The intervention included three discrete components, described below:

\section{Job aid to screen family planning needs}

A job aid, the cover of which is shown in Figure 1, was designed to facilitate the provision of contraceptives upon a woman's request. The job aid is a tool to achieve such improvement and, through brief and targeted questions, prompt the discussion about family planning issues during visits. The job aid had the following components:

A manual entitled How to Provide Direct, Safe, Barrier-free and High Quality Family Planning Services [12]. The manual has three parts. The first directs the provider in screening clients' reproductive intentions and family planning needs, offering contraceptive methods if the woman expresses interest, and facilitating the provision of the method chosen at the time of the visit. The screening is based on three simple questions: (1) would you like to have a child during the next year? (2) if a negative response, are you using a contraceptive method? (3) if a negative response, would you like a method to space or limit childbirth? This instrument is conceptualized as a provider job aid, but it also encourages the client to ask questions about the range of family planning methods available and prompts women to express their unmet needs.

The second part is a test to rule out pregnancy. Providers often did not provide contraceptive methods to clients who were not menstruating at the time of their consultation. This portion of the job aid was designed to rule out a pregnancy and reduce providers' reliance on a policy of providing contraception only when a woman is menstruating. Such test has been accepted by the World Health Organization as a standard procedure to rule out pregnancies [2]. The third part was designed to assist the provider in answering questions and resolving doubts. The checklist includes questions that women commonly ask about methods and evidence-based responses to each. It was developed to help providers discuss fears and questions that women may have.

\section{Training program}

The training program included two main topics as follows:
Logistics training: An 8-hour training program on logistics was provided to staff responsible for supply management at health centers and posts. The purpose of this workshop was to ensure availability of contraceptive supplies at the time of the intervention.

Job aid training: A two-day training program on the use of the job aid and method showcase was conducted for doctors, nurses, nurse auxiliaries and all other service providers who may care for clients at health centers and posts. Research supervisors paid at least one follow-up visit to participating providers following the initial training program, using a previously tested observational checklist to assess the quality of the service provided. The supervision and training checklist documented whether providers asked about their clients' reproductive intentions, and if they provided adequate information and contraceptive methods upon request.

\section{Other support material}

Additional training materials included the legal framework for family planning services in Guatemala, a flipchart describing family planning methods, a presentation of baseline results, a form for requesting family planning supplies, a guide to fill out the family planning supply request form, and the supervisors' observation guide for family planning visits described above. In addition, Ministry of Health personnel who participated in the intervention developed a poster with samples of the different methods offered. The showcase included a brief description of each method and a statement about its use.

Drafts of the job aid were pretested with service providers in and around Guatemala City, and revisions were made based on their experiences and comments, and new drafts were then tested again by other providers, and revisions made based on their experiences and comments.

To make the job aid applicable to monolingual populations nurse auxiliaries were requested to translate when necessary. During training sessions, nurse auxiliaries discussed translation issues. Health Centers and Posts in general have at least one bilingual nurse auxiliary.

\section{Design}

The research design was quasi-experimental with a pretest-posttest-follow-up comparison group design. Health districts, with their health centers and posts, were purposively assigned to intervention or comparison groups to assure comparability of the two groups. The health center staff generally consists of a doctor, a professional nurse, at least one auxiliary nurse, and sometimes other health workers, such as a vaccinator. Each center supervises several health posts, which are smaller and generally staffed by one or more auxiliary nurses. 
This material is to help you deliver contraceptive methods in an easy, safe and quick way with high quality criteria.

Start the conversation asking:

\section{Would you like to have a child during the next year?}

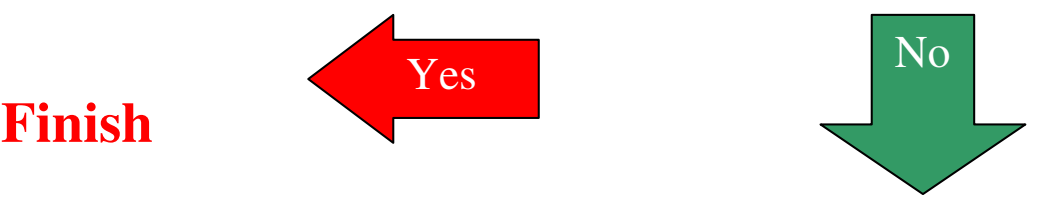

\section{Are you using any method or have you had a sterilization to stop having children?}

Finish
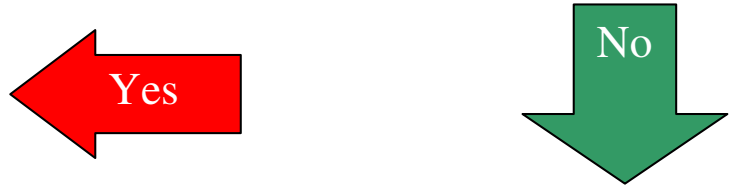

\section{Do you want to use a method to space or limit childbirth?}

Finish

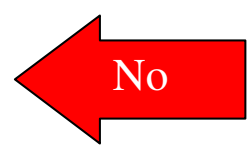

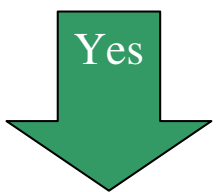

Explain and offer family planning methods

Figure I

Screening family planning needs

The study was conducted in the Department (state) of Sololá. Sololá is one of six departments/health areas that make up the southwest region, where more than $90 \%$ of the population is Mayan. In Solola $44 \%$ of the women of reproductive age have never attended school. Sololá has the second lowest contraceptive prevalence in the Guate- malan highlands. The 1995 National Maternal-Child Health Survey, which provides fertility indices by department, estimated use of any contraceptive method in Sololá at $9.6 \%$ and use of modern methods at $6.0 \%$ for all women of reproductive age married or in union, as compared to national levels of $31.4 \%$ and $26.9 \%$, respectively. 
The Ministry of Health in the Solola Health Area is divided into 10 districts with a total of 10 health centers, 33 health posts, and one national hospital located in the departmental capital. The ten health districts were divided in two groups. There are two districts in the health area that have cities: Sololá and Panajachel. The health center and associated health posts of Sololá were assigned to the intervention group, while those in the Panajachel area were assigned to the comparison group. The other eight districts allowed for similar pairings: two are located on a large lake, two are in the mountains, two are in the lowlands, and two have totally Mayan populations.

The strategy was implemented at intervention sites during a period of six months. Upon completion of the intervention and the post-survey measurements, the former was scaled up to the comparison sites, and a follow-up assessment was conducted nine months after the post-survey in both intervention and comparison groups. Thus the follow-up measurements show the extent to which it was possible to replicate the results obtained during the first phase of the project and the extent to which the changes endured in the intervention sites.

\section{Eligibility criteria and data collection}

Following an informed consent interview, all women who visited a health post or center for whatever reason during a two- to four-day period were screened to identify those who (a) were of reproductive age (15 to 49 years old); (b) were sexually active (proxy: women who declared themselves in union); (c) wished to avoid pregnancy for a 12month period or were not sure if they wanted to get pregnant; and (d) verbally agreed to respond to the questionnaire.

Three rounds of exit interviews were conducted with eligible clients. The baseline was conducted in February 2000, the post-intervention survey was conducted in August 2000, and the follow-up was completed nine months later in May 2001.

\section{Dependent variables}

Dependent variables focused on changes in specific provider practices that limited access to services and quality of family planning information given to clients, regardless of the reason for attending the health services. The exit interviews focused on whether the woman was asked about her reproductive intentions; if she was informed about available methods; if the client was given an opportunity to ask questions; if the provider helped the client to select a method; if a method was provided during the visit or the client was given a referral or appointment for the method (surgical methods are not provided in these facilities); if a method provided was the one the client wanted; and if the provider explained how to use the method.

\section{Results \\ Sample}

A total of 2,552 women were screened for marital status, pregnancy, contraceptive practice, and reproductive intentions to select those who were in union, non pregnant, and did not wish another pregnancy during the following year or were not sure. Of these 27\% (671 cases) were already contracepting at the time of the interview, pregnant, not in union, or wished to become pregnant in the next year, leaving 1,881 women who were exposed to the risk of unwanted pregnancy. These women formed the study sample.

For the baseline survey, 892 women were interviewed over a three-week period, including 451 at intervention health centers or posts and 441 in control sites. The postintervention survey included a total of 480 interviews: 282 at intervention and 198 at control sites. For the follow-up survey, 509 interviews were conducted, 270 at intervention and 239 at control sites.

The principal reason for the visit was to seek care for a sick child, cited by almost half of the women interviewed, followed by care for the woman's own illness, and vaccination for a child. Less than three percent of women in the study reported family planning as their reason for attending the clinic at all three points in time.

The mean age of respondents was 28 . Less than one percent of women interviewed had not had a child at the time of the interview. On average the women had a parity of 4 , with a range of 0 to 16 . No statistical differences were observed in the demographic profiles of the women interviewed in the baseline, post-intervention, and follow-up samples. Thus client samples have comparable demographic characteristics over the three measures.

\section{Screening for family planning needs}

Exit interviews demonstrated that the intervention was instrumental in improving providers' family planning screening practices. As seen in Table 1, providers rarely asked women about their reproductive intentions prior to the intervention ( $7 \%$ at the intervention sites and $10 \%$ at the comparison sites). After the intervention, 37\% of women at risk of unwanted pregnancy at the intervention sites reported they were asked about their reproductive intentions, as compared with $11 \%$ at the comparison sites. The increase was statistically significant. At the ninemonth follow-up, at which time the intervention was being implemented at the comparison sites as well, 30\% of women were asked about their reproductive intentions at intervention sites, while the proportion had doubled $(22 \%)$ at comparison sites after participation in the intervention. (Some decrease at intervention sites is to be expected over time because normal turnover in personnel 
Table I: Percentage distribution of women asked about their reproductive intentions

\begin{tabular}{lllllll}
\hline Treatment group & Baseline & \multicolumn{3}{c}{ Post-intervention } & Follow-Up \\
& $\%$ & $\mathrm{~N}$ & $\%$ & $\mathrm{~N}$ & $\%$ & $\mathrm{~N}$ \\
\hline Intervention * $^{*}$ & 6.7 & 30 & 36.9 & 104 & 29.6 & 80 \\
Comparison** $^{*}$ & 10.0 & 44 & 11.1 & 22 & 22.4 & 53 \\
\hline
\end{tabular}

* Difference from baseline to post-intervention statistically significant at $p \leq .000$ I. ** Difference from post-intervention to follow-up statistically significant at $\mathrm{p} \leq .002$.

Table 2: Percentage distribution of women who were informed about contraceptive methods

\begin{tabular}{lllllll}
\hline Treatment group & Baseline & \multicolumn{2}{c}{ Post-intervention } & Follow-Up \\
\cline { 2 - 7 } & $\%$ & $\mathrm{~N}$ & $\%$ & $\mathrm{~N}$ & $\%$ & $\mathrm{~N}$ \\
\hline Intervention* & 7.8 & 35 & 41.5 & 117 & 35.7 & 96 \\
Comparison ** & 10.0 & 34 & 17.2 & 34 & 31.6 & 75 \\
\hline
\end{tabular}

* Difference from baseline to post-intervention statistically significant at $p \leq .0001$. ** Difference from baseline to post-intervention statistically significant at $\mathrm{p} \leq .008$ and from post-intervention to follow-up t $\mathrm{p} \leq .000$.

brings in providers who have not been exposed to the intervention, job instruments may lose their novelty, or they may wear out or get lost). The final difference between the intervention and the comparison groups is not statistically significant, but the change from the preintervention levels is significant. No significant differences were observed in the demographic characteristics of women who were screened vs. those who were not.

\section{Quality of assistance provided in family planning decision making}

The screening process serves to identify women who may want to space or limit births, potentially all of the women in this study. Therefore, the providers should inform each woman about family planning methods available, provide an opportunity for the woman to ask questions, and help her choose a method if she has difficulty. Finally, the client should receive a method, referral or appointment during the visit if she wants one, and a woman who receives a method should receive the method of her own choice and instructions in its use.

Prior to the intervention, $8 \%$ of eligible women who received services at the intervention sites and $10 \%$ at the comparison sites reported that they had been informed about methods (Table 2). The proportion increased to $42 \%$ at intervention sites post-intervention, compared with $17 \%$ at the comparison sites. The increase was statistically significant at both places. When the intervention was extended to the comparison sites, the proportion of women informed nearly doubled to $32 \%$, which is statistically significant, while the proportion at the intervention sites fell slightly to $36 \%$. It is not clear why there was an increase among the comparison group at the post-intervention. Between the beginning of the study and the postintervention survey, there was a change in national government in Guatemala from one that looked unfavorably on family planning services to one with a favorable policy. This may have begun to have some effect by the time of the post-intervention survey, but such effect is strictly speculative.

Every woman who seeks family planning information or services should be given an opportunity to ask questions after the provider has described the methods available. In this study, prior to the intervention, clients reported that providers rarely gave them an opportunity to ask questions ( $5 \%$ at intervention sites). After the intervention, the proportion of women who reported they had been given an opportunity to ask questions increased to $20 \%$ among the intervention group (Table 3 ). In the comparison sites a similar proportion of women reported at the baseline that they could ask questions (6\%), and this figure doubled to $12 \%$ post-intervention, a statistically significant change that the authors again can not explain. Nine months later at the follow-up, $17 \%$ and $20 \%$ of clients at the intervention and comparison sites respectively reported having an opportunity to ask questions, a signif- 
Table 3: Percentage distribution of women who had an opportunity to ask questions

\begin{tabular}{llllll}
\hline Treatment group & Baseline & & Post-intervention & Follow-UP \\
\cline { 2 - 6 } & $\%$ & $\mathrm{~N}$ & $\%$ & $\mathrm{~N}$ & $\%$ \\
\hline Intervention* & 5.1 & 23 & 20.2 & 57 & 17.4 \\
Comparison** & 5.9 & 25 & 11.6 & 23 & 47 \\
\hline
\end{tabular}

* Difference from baseline to post-intervention statistically significant at $\mathrm{p} \leq .000 \mathrm{I}$. ** Difference from baseline to post-intervention statistically significant at $\mathrm{p} \leq .012$ and baseline to follow-up at $\mathrm{p} \leq .000 \mathrm{I}$.

Table 4: Percentage distribution of women who reported receiving assistance in selecting a method

\begin{tabular}{lllllll}
\hline Treatment group & Baseline & \multicolumn{2}{c}{ Post-intervention } & Follow-Up \\
\cline { 2 - 7 } & $\%$ & $\mathrm{~N}$ & $\%$ & $\mathrm{~N}$ & $\%$ & $\mathrm{~N}$ \\
\hline Intervention* & 4.4 & 20 & 13.1 & 37 & 11.5 & 31 \\
Comparison** & 5.0 & 21 & 4.0 & 8 & 16.9 & 40 \\
\hline
\end{tabular}

* Difference from baseline to post-intervention statistically significant at $p \leq .000$ I. ** Difference from post-intervention to follow-up statistically significant at $\mathrm{p} \leq .000 \mathrm{I}$.

Table 5: Percentage distribution of women who reported receiving a method, appointment or referral

\begin{tabular}{|c|c|c|c|c|c|c|}
\hline \multirow[t]{2}{*}{ Treatement group } & \multicolumn{2}{|c|}{ Baseline } & \multicolumn{2}{|c|}{ Post-intervention } & \multicolumn{2}{|c|}{ Follow-Up } \\
\hline & $\%$ & $\mathrm{~N}$ & $\%$ & $\mathrm{~N}$ & $\%$ & $\mathrm{~N}$ \\
\hline \multicolumn{7}{|c|}{ Received a Method only } \\
\hline Intervention * & 0.4 & 2 & 9.9 & 28 & 4.8 & 13 \\
\hline Comparison $* *$ & 0.9 & 3 & 0.5 & 1 & 3.0 & 7 \\
\hline \multicolumn{7}{|c|}{ Received a Method, Appointment or Referral } \\
\hline Intervention $* * *$ & 3.1 & 14 & 17.4 & 49 & 18.5 & 50 \\
\hline Comparison & 5.2 & 23 & 6.6 & 13 & 9.2 & 22 \\
\hline
\end{tabular}

$*$ Difference from baseline to post-intervention statistically significant at $p \leq .000 \mathrm{I}$. **Difference from baseline to follow-up statistically significant at $\mathrm{P} \leq .044$. *** Difference from baseline to post-intervention statistically significant at $\mathrm{p} \leq .000 \mathrm{I}$.

icant change for the comparison group after participating in the intervention.

After a woman has received information on the methods available and asked any questions she may have, the next step is for her to select a method if she wants one. Many rural, indigenous women in Guatemala have little or no experience with modern methods of family planning. In previous studies the researchers had noticed that some women needed additional help in making a decision after receiving information about methods available. The providers at the intervention sites were trained to ask the woman a series of question following a decision-making tree in the manual. The provider first asks who should be the user of the method, the woman or her husband. Then the provider asks if she wants to end childbearing or space the next birth. If she does not want any more children, the provider is directed to explain first a surgical option depending on whether the woman wants to use it or prefers her husband do so. If the woman wants to space a subsequent birth or does not want a surgical method, she is informed of pills, injections, IUDs, and natural methods if she wants a method for herself, and condoms if she prefers a method for her husband. Before the intervention, less than $5 \%$ of clients reported having received any assistance on the part of the provider in making a choice 
(Table 4). After the intervention, $13 \%$ of women at risk of unwanted pregnancies at the intervention sites reported receiving assistance from providers, while women reported no increase at comparison sites. Comparison sites, however, show levels comparable to the intervention group after implementation of the intervention $(17 \%)$.

Perhaps the most challenging behavioral variable that this study aimed to improve was the actual delivery of methods at the time of the visit. Prior to the study, less than one percent of all women at risk of an unwanted pregnancy reported they received a family planning method during their visit to the health post or center. Post-intervention, $10 \%$ of at-risk clients seen at intervention sites reported receiving a method during the visit, although this figure later decreased to $5 \%$ (top of Table 5). At comparison sites, there was no change at the mid-term, and the proportion rose to $3 \%$ after these providers were trained in the intervention, which was statistically significant.

Since some methods can not be provided in these settings, they require a future appointment or referral. For example, none of the study sites is suitable for delivery of surgical methods. Since the women in this study had an average of 4 living children, it is likely that some expressed interest in ending childbearing. Thus it seemed reasonable to consider a referral or future appointment as a desirable behavior change on the part of providers.

At baseline 3\% of women in the intervention group received a method, referral or appointment. This figure increased to $17 \%$ post-intervention and $19 \%$ nine months later at the follow-up (bottom Table 5). The change was statistically significant. The picture was less clear among the comparison group. At baseline and post-intervention, $5 \%$ and $7 \%$ of women in the comparison group reported receiving a method, referral or appointment. However, at the follow-up, there was still no statistically significant increase (9\%). Researchers are unable to explain why providers in comparison sites failed to provide methods, appointment or recommendations, especially considering their improvement in all the rest of the screening process. A possible reason is unavailability of methods among comparison sites, but such information was not collected during the study.

The intervention was designed to focus the screening on the individual woman's preferences. It was important, therefore, to assess the extent to which a woman who received a method received the contraceptive of her choice and whether the provider explained the method's use to the woman's satisfaction. It was equally important for the researchers to assure that women were being assisted, not pressured, to select a method.
All women except one in the intervention group at the time of the post-intervention survey reported they had received the method they desired, and all but one in the same group reported receiving adequate instruction in the use of the method. However, the number of women who received a method was too small to be statistically meaningful.

\section{Comparison of professional and auxiliary performance}

In this study doctors provided $13 \%$ of the services, nurses $7 \%$, auxiliary nurses $73 \%$, and a mix of other health care workers, $7 \%$. Informal interviews with doctors during the course of the study gave researchers reason to suspect that some of the doctors might not use the manual following training. Some doctors expressed a fear that making reference to a manual would lead to a clients' lack of confidence in the doctor's knowledge, and in fact the results suggest that doctors may have used their training and the manual infrequently if at all.

Auxiliary nurses significantly improved their screening behaviors, asking about reproductive intentions and describing methods available. Physicians did not display a pattern of improvements, and nurses often had too few examples of positive behavior to apply statistical tests.

When it came to allowing for questions, there was no consistent pattern. The auxiliary nurses in the intervention group improved between the baseline and post-intervention, while the comparison group did not. There was no statistically significant change in either group between the post-intervention survey and the follow-up.

When it came to assisting a woman to select a method, the pattern was clearer. There was a statistically significant improvement only in the intervention group at the postsurvey, and there was a statistically significant improvement in the comparison group after they participated in the intervention.

When it came to providing a method, appointment or referral, the intervention group of auxiliary nurses improved from $4 \%$ to $22 \%$ at the post-survey, while there was no statistically significant change among the comparison group. However, there was no change in the comparison group at the follow-up, while the intervention group remained at the same level.

\section{Discussion}

This study was designed for a very conservative environment in which women have reduced access to family planning information and services because providers do not readily offer them and women are reluctant to request them. The study tested an intervention to improve the role 
of the provider in offering services and giving women a better opportunity to request them with some success.

The research intervention was most effective in improving screening practices. The greatest changes were seen in the proportion of providers who screened women for their reproductive intentions and informed them about the family planning methods available. This first step is a prerequisite for further progress in making services more widely available in the public sector. The results of this study have received attention from the Ministry of Health of Guatemala since 2000 when a new administration came to power that is favorable to delivery of family planning services. The screening procedures, which demonstrated the greatest improvement, are now included in the training of all reproductive health service providers in the country.

\section{Conclusions}

There were moderate improvements in increasing women's opportunities to raise questions during their health care visits, providers' assisting women in making their choices, and increasing delivery of a family planning method, referral or appointment during a visit. Further research into new strategies is needed in these areas.

Since women in both intervention and comparison groups generally reported having received their preferred method, it appears that the study avoided the possible pitfall in which providers might become overzealous and guide women to a method preferred by the provider rather than to the woman's individual preference.

Although post-intervention provision of a contraceptive remains low, the intervention is useful because it represents a first step towards effective contraceptive delivery in an environment where active screening and offering of contraceptive education and methods have traditionally been limited. The job aid helped the provider easily identify if the woman might need a contraceptive method or would like to receive more information. Prompted by key questions, women are placed in a position to ask further questions about contraceptives and choices. Such client empowerment is a key element to break cultural barriers to family planning.

\section{Competing interests}

None declared.

\section{Authors' contributions}

This study was designed and supervised between December 2000 and July 2001 by Marianne Burkhart, Director of the Population Council office in Guatemala at the time of the study. Francisco Mendez and Felipe Lopez were responsible for project implementation and Carlos Bram- bila was responsible for the follow-up phase of the research project.

\section{Acknowledgements}

This project was conducted with the support of USAID/G-CAP under Cooperative Agreement No. 520-0357-A-00-4169-00 with the Population Council, which is gratefully acknowledged. The collaboration of colleagues and partners from the Ministry of Health, Sololá, Guatemala made this project possible.

\section{References}

I. Shelton J, Angle M, Jacobstein R: Medical barriers to access family planning. Lancet 1992, 340:1334-35.

2. Stanback J, Thompson A, Hardee K, Janowitz B: Menstruation requirements: a significant barrier to contraceptive access in developing countries. Studies in Family Planning 1997, 28:245-50.

3. Bertrand JT, Hardee K, Magnani RJ, Angle MA: Access, quality of care and medical barriers in family planning programs. International Family Planning Perspectives 1995, 21:64-9.

4. POLICY Project: Un estudio de barreras médicas en Guatemala [A study on medical barriers in Guatemala]. Guatemala City, Guatemala, POLICY Project 1999.

5. Vernon R, Ottolenghi E, Leon F: Oferta sistemática de servicios de planificación familiar y salud reproductiva en Guatemala. [Systematic provision of family planning and reproductive health services in Guatemala]. INOPAL final report. Guatemala City, Guatemala, Population Council 1998.

6. Leon FR: Estrategia de consejería balanceada para mejorar la calidad de atención en planificación familiar en Es/Salud/ Lima [Balanced counseling strategy to improve family planning care at ES/Salud/Lima]. Frontiers Final Report. Lima, Peru, Population Council 2002.

7. Amendola L, Euceda D, Lundgren R, Mendoza I, Ali N: El uso de listados para detectar y referir mujeres de comunidades rurales a servicios de salud reproductiva. [The use of checklists to screen and refer women from rural communities to reproductive health services]. INOPAL final report. Tegucigalpa, Honduras, Population Council 1998.

8. Instituto Nacional de Estadística (INE): Guatemala: Encuesta nacional de salud materno-infantil [Guatemala: National maternal and health survey]. Guatemala City, Guatemala, Instituto Nacional de Estadística 1995.

9. Instituto Nacional de Estadística (INE): Guatemala: Encuesta nacional de salud materno-infantil 1999 [Guatemala: National maternal and child health survey 1999]. Guatemala City, Guatemala, Instituto Nacional de Estadística 1999.

10. Instituto Nacional de Estadística (INE): Encuesta nacional de ingresos familiares (ENIGFAM) 1998-1999 [National family income survey: 1998-1999]. Guatemala City, Guatemala, Instituto Nacional de Estadística 1999.

II. Ministerio de Salud Pública y Asistencia Social Instituto Nacional de Estadística, Center for Disease Control and Prevention (CDC): Encuesta nacional de salud materno-infantil 2002. [National maternal and child health survey: 2002]. Guatemala City, Guatemala, Instituto Nacional de Estadística 2002.

12. Burkhart M, Mendez F, Lopez F: How to provide direct, safe, barrier-free and high quality family planning services. Guatemala City, Guatemala: Population Council 1999.

\section{Pre-publication history}

The pre-publication history for this paper can be accessed here:

http://www.biomedcentral.com/1472-698X/4/2/prepub 